\title{
Promoting ethical follower behaviour through leadership of ethics: The development of the ethical leadership inventory (ELI)
}

\author{
H. Spangenberg* \\ Centre for Leadership Studies, Graduate School of Business, \\ University of Stellenbosch, Stellenbosch 7600, Republic of South Africa \\ hspan@usb.sun.ac.za \\ C.C. Theron \\ Department of Industrial Psychology, University of Stellenbosch, \\ Stellenbosch 7600, Republic of South Africa \\ Received December 2004
}

\begin{abstract}
The Centre of Leadership Studies developed a model that defines and describes leadership behaviour required for creating an ethical and high performing organisation. Purposefully building an ethical organisational environment is a prerequisite for ethical organisational behaviour. To be an ethical high performance leader, a person must be both an effective leader and a leader of ethics. The purpose of this study is to develop a $360^{\circ}$ instrument that can be used to assess the quality of ethical leadership of middle, senior and executive managers in public, private and not-for-profit organisations. The results reported here provide reasonable support for the use of the ELI. The possibility of causal influences existing amongst specific first-order leadership dimensions, however, needs to be investigated.
\end{abstract}

*To whom all correspondence should be addressed.

\section{Introduction}

The organisational misbehaviour as reported in daily newspapers and academic journals in the USA towards the end of the 1980's, lead to the observation that people have lost faith in the values of their economic society and that they needed a spiritual rebirth in business leadership. The question arose whether industry needed a new religion, or at least a better one than it has had (Ohmann, 1989). Reflecting on this premise, Kanungo and Medonca (1996) argue that a business corporation in itself is incapable of doing good or evil in society. Such outcomes are due entirely to the unethical behaviour of individuals, whether it is employees or managers. Although all employees are responsible for achieving organisational objectives in an ethical manner, the accountability for providing proper direction and high performance standards lies with organisational leaders. In the South African context, where reports on corruption and corruption-related incidents in public, business, sports, and other organisations regularly form part of the major evening news bulletins of SABC, the official broadcaster, the same reasoning applies. The duty and responsibility for providing ethical high performance leadership rests with executive and senior managers of South African business, public service, non-governmental, sports and other leisure organisations.

The King Report on Corporate Governance in South Africa (King Committee on Corporate Governance, 2002:101) defines organisational ethics as 'the principles, norms and standards that it promotes for the guidance and conduct of its activities, in accordance with established values'. The
King Report further contends that adherence to established principles of ethical conduct provides a strong measure of organisational integrity and states that 'a company's ethical principles represent a major motivator of stakeholder involvement with it and, as such, should permeate its culture, motivating its strategy, business goals, policies and activities' (p.102).

With regard to the implementation of corporate ethics, Andrews (1989:99) describes three challenges that organisations face, namely 'the development of the executive as a moral person; the influence of the corporation as a moral environment; and the actions needed to map a high road to economic and ethical performance - and to mount guardrails to keep corporate wayfarers on track'. Bass and Steidlmeier (1999) propose three pillars of ethical leadership that support the importance of the ethical development of the organisational leader and further build out Andrews's challenges. The pillars are the moral character of the leader and his/her concerns for self and others, ethical values being embedded in the leader's visioning, and the morality of the choices and actions of leaders and their followers.

Jose and Thibodeaux (1999), quoting research, describe a number of specific roles for the ethical leader:

1. With regard to visioning, they suggest that leaders set the moral standards for the organisation and focus on the integrity of the common purpose (Hosmer, 1987). 
2. Leaders explicitly clarify the ethical dimensions of management decisions, and formulate and justify ethical principles that govern decision-making (Enderle, 1987).

3. Leaders signal their commitment to ethical principles through their influence on corporate culture.

- They do this by defining organisational ethics and values, and by acting as role models in establishing a positive ethical climate. Employees normally receive their ethical guidance from observing the behaviours of their superiors (Jose \& Thibodeaux, 1999; O`Boyle \& Dawson, 1992).

- Leaders build culture by what they monitor and control, how they react to critical events and what criteria they use for recruiting, selecting, rewarding and dismissing organisational members (Schein, 1992).

- In addition to a strong impact on the ethical behaviour of employees, ethical leaders facilitate trust (Brien, 1998).

4. The CEO faces the challenge of creating a high degree of congruence between the organisations's guiding beliefs and employees' every-day beliefs (Jose \& Thibodeaux, 1999).

In order to describe leadership required for creating an ethical and high performing organisation and to put it in proper organisational context, a theoretical model was developed. The model comprises four phases. It is proposed that the ethical orientation of leaders (Phase 1); exemplified by building an ethical organisation, i.e. applying transformational influencing processes, building of ethical structures, creating an ethical culture and climate, and developing productive stakeholder relations (Phase 2); mediated by external factors (Phase 3); will create an ethical organisational environment and positive outcomes for the organisation, its employees and other stakeholders (Phase 4).

Phase 2 of the model, namely 'Building an ethical organisation' served as theoretical basis for the development of the leadership of ethics assessment instrument. The first component of building an ethical organisation is applying transformational influencing strategies. This broad construct can be equated with the core leadership processes of analysing the organisation's external and internal environments, developing and communicating the vision, preparing the people and the organisation for implementing the vision and, finally, implementing the vision and strategy (Conger \& Kanungo, 1998; House, 1995; Kanungo \& Medonca, 1996; Spangenberg \& Theron, 2002). The other three elements of Phase 2, namely creating ethical structures (Brickley, Smith \& Zimmerman, 1994; James, 2000; Lindsay, Lindsay \& Irvine, 1996; Trevino \& Nelson, 1995); creating an ethical culture and climate (Cullen, Victor \& Bronson, 1993; Deshpande, 1996; Trevino \& Butterfield, 1998; Upchurch \& Ruhland, 1996; Vaicys, Barnett \& Brown, 1996; Vardi, 2001; Victor \& Cullen, 1987; 1990); and building productive stakeholder relationships Hummels,
1998; Husted, 1998; Maignan \& Ferrell, 2000; Strong, Ringer \& Taylor, 2001) are also key responsibilities of the leader of ethics.

During the development of the model it was realised that ethical leadership comprises an important but separate dimension of leadership. While the aims of a traditional organisational vision is directed mainly at overall organisational and people performance and effectiveness, an ethical vision is aiming at achieving ethical organizational behaviour. An ethical vision may include imperatives such as, for example, giving people a sense of moral purpose, reconciling business efficiency with social responsibility, fostering the uniqueness and integrity of the individual and group, and treating all internal and external stakeholders with respect, honesty and integrity.

In order to create an ethical high performance organisation, it is suggested that the creation and application of an ethical vision could either form part of the overall organisational visioning process, or run parallel but in concert with the larger visioning process. Ethical leadership can thus be operationalised and measured in terms of the same process model on which the Leadership Behaviour Inventory (LBI) is based (Spangenberg \& Theron, 2002). In terms of this model, leadership of ethics comprises the creation and sharing of an ethical vision (based on a careful diagnosis of the external and internal environments in which all relevant parties participate); preparing the leader, followers, and the organisation - particularly its structures and culture - for implementing the vision; and the actual implementation process itself. This observable, behaviourally-based leadership process lends itself readily to behaviour assessment by means of $360^{\circ}$ methodology.

An issue that needs to be clarified is the question whether the person who does well in a leadership assessment questionnaire [that normally would include some ethical leadership dimensions (for example the $\mathrm{LBI}^{1}$ )] can be considered a leader of ethics? One could probably answer such a question by arguing that such a person could be considered an honest leader with integrity who creates trust in the work unit and in him/herself. However, to be an ethical high performance leader, a person must be both an effective leader (as per LBI or $\mathrm{MLQ}^{2}$, for example) and a leader of ethics. In essence, the LBI predicts that an individual will display leadership effectiveness (measured by a large variety of dimensions) and ethical behaviour (measured by a few important ethical dimensions). However, to enact leadership of ethics, a person must purposefully and consciously demonstrate and cultivate ethical behaviour in his/her work unit. The leader must, therefore, purposefully facilitate the development of an ethical organisational culture by instilling and cultivating

\footnotetext{
${ }^{1}$ The Leadership Behaviour Inventory (LBI), measures a four-phase process of leadership (Spangenberg \& Theron, 2002), including transformational leadership dimensions and a number of dimensions that have a distinct ethical content, e.g. enabling the leader (two dimensions) enabling followers, interpersonal relations, concern for others, and character-related dimensions such as honesty and integrity, and decisiveness and hardiness.
}

${ }^{2}$ Multi-factor Leadership Questionnaire (Bass \& Avolio, 1997). 
ethical beliefs and values (Trevino, Hartman \& Brown, 2000). To conclude this argument, the King Report (2002:106) states that 'the moral objectives of the company, exemplified by the board collectively and the directors individually, needs to be made visible to both internal and external stakeholders and seen to be integral with other corporate objectives'.

Since leadership of ethics is a crucial force in creating an ethical organisation, it was decided to first develop an ethical leadership inventory, to be followed by the development of an ethics audit for organisations at a later stage. By assessing the quality of the leadership of ethics of members of a management team on a $360^{\circ}$ basis, insight will be increased considerably into their own ethical leadership behaviour as well as understanding of the entire domain of leadership of ethics and the impact thereof on their units. Greater self-awareness and increased understanding of ethics in organisational dynamics will provide a positive climate for major individual and team learning initiatives.

The purpose of this study is, therefore, to develop a $360^{\circ}$ instrument that can be used to assess the quality of leadership of ethics of middle, senior and executive managers in public, private and not-for-profit organisations. In terms of the foregoing argument it probably would have been appropriate to refer to such an instrument as the Leadership of Ethics Inventory. Informal consultation with leaders participating in the study, however, indicated that market acceptance of the instrument would most likely be better if the more conventional title Ethical Leadership Inventory (ELI) would be adopted.

\section{Development of the questionnaire}

\section{Strategy for developing the questionnaire}

1. Generation of items. The main source of items was the literature on the development of ethics in organisations. A second source of items was the Leadership Behaviour Inventory (LBI). A significant number of items from the LBI research copy, consisting of 237 items, had ethical content and with minor adaptations could be included in the ELI research copy. Research indicates that LBI items measure observable leadership actions and behaviour with a high degree of reliability and validity (Spangenberg \& Theron, 2002).

2. Using the structure of the Leadership Behaviour Inventory (LBI) as a basis for classifying newly developed items. The rationale was that the LBI comprises four actionable phases that are required for implementing a vision, irrespective of whether it is a performance or ethics-related vision.

3. Using a Delphi technique in field research. This means that at critical stages in the development process, a panel of experts verify the process and propose improvements as required. Positive experience on three occasions in the past of using the Delphi technique as an aid in questionnaire development has suggested that this iterative, consensus seeking technique should be employed again (Spangenberg \& Theron, 1997; 2002; Theron \& Spangenberg, 2004).

\section{Field research for developing the questionnaire}

Two rounds of field research by means of the Delphi technique were conducted. During the first round the draft questionnaire was sent to a panel of 13 industrial psychologists, known as experts in the field of leadership assessment and/or development. They were asked to work through the questionnaire as follows: verify relevance of dimensions and propose new dimensions if required; rate the appropriateness of each item on a three-point scale, and propose new items as required.

The first draft questionnaire consisted of 24 dimensions, measured by means of 276 items. In order to provide a better understanding to raters, dimension definitions were included and items were categorised according to dimensions. On return of questionnaires a two-stream approach was followed to improve or cull items. Firstly, in line with the Delphi technique, comments on specific dimensions and items were studied. Comments from raters were indeed extremely useful in improving items. Secondly, descriptive statistics were conducted on item ratings and the culling of items was based mainly on arithmetical decision rules. The improved and shortened draft questionnaire still consisted of 24 dimensions, but the number of items was reduced to 189 .

It was decided to conduct a second round of field research by means of the Delphi technique in which organisational leaders would act as raters. It was realised that in order to facilitate sound corporate governance that simultaneously increases ethical, high organisational performance and decreases organisational malpractices, the support and continued involvement of a significant body of high-level organisational leaders would be required. Franklin Sonn, former South African ambassador to the USA and prominent businessman, in a personal discussion (May 2003), put it bluntly that an ethical leadership instrument developed by an institution of learning, without consent and support of the leadership community, will not have the desired effect.

The second round Delphi was conducted along the same lines as the first one, with 16 executive managers acting as raters. The group included CEO's from some of South Africa's largest and most respected companies. Ratings and rater comments were very useful in reducing dimensions by collapsing them into other dimensions or by culling them. Ratings and rater comments were again useful in culling items that received unsatisfactory ratings, and to further improve items that were retained. The draft questionnaire was reduced to 19 dimensions, measured by 103 items. The dimensions measured by the ELI, with their definitions, are presented in Table 1. 
Table 1: First-order latent leadership dimensions measured by the ELI

\author{
1. Creating and sharing an ethical vision \\ Understanding the ethical dynamics in the external and internal environments (Environ) \\ Diagnoses ethical dynamics in the external and internal environments in order to develop an ethical vision. \\ Developing a challenging vision (Evision) \\ Develops a collective ethical vision that inspires people and gives them a sense of purpose, is customer-focused and advances \\ diversity of people. \\ Building trust in the leader and the unit (Butrust) \\ The leader creates trust in him/herself and builds confidence in the unit. \\ Articulating an ethical vision and enlisting followers (Artivis) \\ Articulates an ethical vision for the future that provides direction. Inspires confidence in the vision and obtains follower \\ commitment to the vision. \\ Conceptualising ethical strategy (CoStrat) \\ Defines strategic ethical issues clearly. Builds strategies and plans based on thorough problem analysis and broad-based fact- \\ finding. Considers consequences of decisions.
}

\title{
2. Enabling the leader and the unit to implement the ethical vision
}

Enabling the leader (Enablel)

Identifies challenging opportunities for self-development and is committed to continuous learning. Appreciates feedback and has good insight into own his/her own ethical identity, capabilities and behaviour. Is committed to continuous learning.

Empowering followers (Enablef)

Encourages followers to accept responsibility for their own ethical learning and growth. Creates conditions which allow them the opportunity to take meaningful decisions.

Formulating and implementing ethical structures and systems (Strusys)

Adapts structures, processes and procedures to support implementation of ethical strategy in a changing environment.

Implements ethical structures and systems, for example a code of ethics, an ombudsman, ethics committee, and ethics training programme.

Building an ethical culture and climate (Ethcult)

Builds a culture that reflects shared beliefs, values and norms; shared perceptions of ethically correct behaviour;and guidance for handling difficult ethical issues.

\section{Implementing the ethical vision}

\subsection{Leading with courage, integrity and sensitivity}

Acting honestly and with integrity

Honestly manages the organisational unit and consistently lives out the values embedded in the vision (Hointeg)

Considers ethical implications of decisions, assures agreed upon values are adhered to and deals honestly with all stakeholders.

Decisiveness and hardiness (Actdeci)

Acts decisively and makes tough ethical decisions. Performs effectively under stress and reacts positively to change and

uncertainty.

Demonstrating interpersonal sensitivity (Demsens)

Considers the needs, feelings and dignity of others. Works towards productive interpersonal relations..

\subsection{Encouraging ethical behaviour}

Challenging current reality and stimulating learning (Challen)

Challenges current thinking about ethics, reconsiders and improves current practices on an ethical basis. Promotes continuous ethical learning.

Inspiring people towards ethical behaviour (Inspire)

Raises the aspirations of followers and builds confidence in them to perform effectively and ethically. Articulates ethical issues clearly.

\subsection{Stimulating ethics across boundaries}

Facilitating interdepartmental co-ordination (Intcoor)

Facilitates cross-functional collaboration and teamwork. Helps people to see the ethical big picture.

Influencing external stakeholders (Infstak)

Maintains productive relationships with external stakeholders and builds the ethical image of the organisation.

\subsection{Leading ethical initiatives and rewarding ethical contributions}

Planning and implementing ethical initiatives (Planimp)

Ensures that ethical expectations of the unit and its members are clarified, and that ethical initiatives are designed and aligned with ethical and business strategies.

Reviewing ethical initiatives and behaviour (Reviewe)

Reviews the outcomes of unit, team and employee ethical initiatives. Provides specific feedback to followers in order to help them assess their own contribution to these initiatives.

Rewarding ethical contributions and behaviour (Rewarde)

Gives recognition for accomplishing ethical initiatives as well as for exemplary work-related attitudes and behaviour; celebrates ethical success. 


\section{Field testing of the questionnaire}

In order to ensure that the sample reflects diversity of culture and organisational type, a wide range of organisations were approached for participation in fieldwork. Organisational co-ordinators facilitated the nomination of candidates (participants) and respondents. Firstly, they identified colleagues who managed work units, which were defined as consisting of three or more members, with no upper limit. Co-ordinators were specifically asked to include unit managers from all cultures, male and female, in order to obtain a representative sample. Candidates completed a Candidate Questionnaire and were promised to receive personal feedback on request.

Secondly, the candidate, in conjunction with the organisational co-ordinator, ideally identified two peers and two subordinates or followers as respondents. The superior of the candidate served as fifth respondent. Respondents completed a Respondent Questionnaire for the candidate. Again, co-ordinators were specifically asked to include a representative sample of respondents. In order to conduct test bias studies on the questionnaire, both candidates and respondents were asked to provide essential biographical information.

\section{Sample}

The data used for the purpose of this study was obtained by means of a series of non-probability samples of unit leaders selected from a non-probability sample of organizations. A total of sixty unit leaders were selected and rated $360^{\circ}$ by their superiors, peers and subordinates. The samples of respondents that rated each selected unit leader again were selected through non-probability sampling procedures. Selected unit leaders also rated themselves, which resulted in a total of 315 completed ELI questionnaires at the time of the analysis. Although the objective initially was to obtain ratings from two subordinates, two peers and a single superior, the need for a large as possible sample size, in conjunction with the difficulties encountered when trying to apply a questionnaire of this length and nature to respondents on this high a job level, necessitated a deviation from this ideal in a number of cases.

The sample of organizations and the number of unit leaders sampled from each organization is depicted in Table 2.

Table 2: distribution of unit leaders across sampled organisations

\begin{tabular}{lcrcc}
\hline \multicolumn{1}{c}{ Company } & Frequency & Percent & $\begin{array}{c}\text { Valid } \\
\text { Percent }\end{array}$ & $\begin{array}{c}\text { Cumulative } \\
\text { Percent }\end{array}$ \\
\hline Anglo Gold & 3 & 5,0 & 5,0 & 5,0 \\
Distell & 4 & 6,7 & 6,7 & 11,7 \\
Medi- Clinic & 4 & 6,7 & 6,7 & 18,3 \\
Peninsula & 7 & 11,7 & 11,7 & 30,0 \\
Technikon & & & & \\
Power Group & 7 & 11,7 & 11,7 & 41,7 \\
Rand Water & 10 & 16,7 & 16,7 & 58,3 \\
SA Reserve Bank & 10 & 16,7 & 16,7 & 75,0 \\
SAPS & 14 & 23,3 & 23,3 & 98,3 \\
Telkom S A & 1 & 1,7 & 1,7 & 100,0 \\
Total & 60 & 100,0 & 100,0 & \\
\hline
\end{tabular}

The distribution of unit leaders in terms of position level is portrayed in Table 3 .

The breakdown of the sample of unit leaders in terms of race is given in Table 4. A pleasing feature of the race composition of the sample is that almost $50 \%$ of the unit leaders belong to previously disadvantaged groups. Unit leaders from previously disadvantaged groups do, however, tend to occupy the relatively lower position levels (cross tabulation not shown).

A stronger representation of unit leaders in the top management category (that is unit leaders responsible for larger, composite units) would have been preferable. In the final analysis it is these higher echelon leaders that should be held accountable for the ethical behaviour of all their followers. It is their ethical vision that should be cascading down through the organizational hierarchy, carried by the walk and talk of lower level unit leaders.

The descriptive statistics characterizing the age distribution of the sample of unit managers are shown in Table 5. Results indicate an approximately symmetric, mesokurtic age distribution.

Due to the sampling methodology and the sample size, the sample can clearly not claim to constitute a representative section of the population of South African unit leaders who could be held accountable for the ethical behaviour of the members of their units. This precludes the possibility of reaching any definitive conclusion on the competence of South African unit leaders on the leadership competencies serving the formulation and realization of an ethical unit vision. This also rules out the possibility of reaching any definitive conclusion on the ability of the ELI to successfully measure the leadership of ethics construct in accordance with the instrument architecture in the target population. Nonetheless, if the measurement model implied by the instrument design would fit the sample data well, it would constitute relevant, albeit limited evidence that the ELI can be used to assess ethical leadership competencies for development in the target population.

\section{Missing values}

Missing values presented a problem that had to be addressed before analysis could proceed. The same arguments as those presented in Theron and Spangenberg (2004) were considered with regards to the missing values problem. Pair-wise deleting of cases presented itself as a possible solution in this case since it would not result in a correlation matrix with extreme variation in $\mathrm{N}$-values (a maximum of 307 and a minimum of 221 in this particular case). The PRELIS program (Jöreskog \& Sörbom, 1996a) was used to impute missing values. The PRELIS run proved to be reasonably effective in countering the missing value problem in comparison to the results that would have been achieved under list wise deletion, but less useful in comparison to the results that would have been obtained under pair-wise deletion. By default, cases with missing values after imputation were eliminated. After imputation, 264 cases with observations on all 103 items remained in the validation sample. 
Table 3: Distribution of unit leader position level

\begin{tabular}{|c|c|c|c|c|c|}
\hline & Position level & Frequency & Percent & Valid Percent & Cumulative Percent \\
\hline \multirow[t]{4}{*}{ Valid } & Top management & 9 & 15,0 & 18,8 & 18,8 \\
\hline & $\begin{array}{l}\text { Senior management/ } \\
\text { senior professional }\end{array}$ & 23 & 38,3 & 47,9 & 66,7 \\
\hline & $\begin{array}{l}\text { Middle management/ } \\
\text { professional }\end{array}$ & 16 & 26,7 & 33,3 & 100,0 \\
\hline & Total & 48 & 80,0 & 100,0 & \\
\hline Missing & System & 12 & 20,0 & & \\
\hline Total & & 60 & 100,0 & & \\
\hline
\end{tabular}

Table 4: Distribution of unit leader race

\begin{tabular}{|c|c|c|c|c|c|}
\hline & Race & Frequency & Percent & Valid Percent & Cumulative Percent \\
\hline \multirow{5}{*}{$\overline{\text { Valid }}$} & Asian & 3 & 5,0 & 5,9 & 5,9 \\
\hline & Black & 13 & 21,7 & 25,5 & 31,4 \\
\hline & Coloured & 8 & 13,3 & 15,7 & 47,1 \\
\hline & White & 27 & 45,0 & 52,9 & 100,0 \\
\hline & Total & 51 & 85,0 & 100,0 & \\
\hline Missing & System & 9 & 15,0 & & \\
\hline Total & & 60 & 100,0 & & \\
\hline
\end{tabular}

Table 5: Descriptive statistics characterizing the unit leader age distribution

\begin{tabular}{lc}
\hline Descriptive statistics & Value \\
\hline N Valid & 52 \\
N Missing & 8 \\
Mean & 43,25 \\
Median & 43,00 \\
Mode $^{\mathrm{a}}$ & 41 \\
Std, Deviation & 7,987 \\
Variance & 63,799 \\
Skewness &, 061 \\
Std, Error of Skewness &, 330 \\
Kurtosis &,- 138 \\
Std, Error of Kurtosis &, 650 \\
Minimum & 25 \\
Maximum & 62 \\
\hline
\end{tabular}

a Multiple modes exist. The smallest value is shown

The relative narrow band in which the effective sample sizes vary under pair-wise deletion, combined with the fact that the median effective sample size is 275 and the modal effective sample size 278, swayed the decision towards the use of pair-wise deletion of missing values. The effective sample size that has been obtained through imputation by matching falls below the twenty-fifth percentile $\left(\mathrm{P}_{25}=267\right)$.

\section{Item analysis}

The architecture of the ELI reflects the intention to construct essentially one-dimensional sets of items to reflect variance in each of the nineteen latent variables collectively comprising the ethical leadership domain. The items are meant to function as homogenous stimulus sets to which raters respond with behaviour that is primarily a relatively uncontaminated expression of a specific underlying ethical leadership latent variable. Each of the nineteen ELI subscales were consequently item analysed through the SPSS Reliability Procedure (SPSS 11.0 for Windows, 2004) to identify and eliminate items not contributing to an internally consistent description of the unit performance facet in question. The summary results of the item analyses are shown in Table 6.

Two items were flagged as problematic. Item 3 of the Building trust in the leader and the unit subscale and item 48 of the Acting honestly and with integrity subscale were identified as items that lower the homogeneity of the scales. The magnitude of the corrected item-total correlations $(0,2091$ and 0,3598$)$ and the increase in alpha affected by the removal of the items $(0,1391$ and 0,0309$)$ justified the deletion of these items. Four further items (items 1, 4, 13 and 54) were flagged as suspect. The magnitude of the corrected item-total correlation $(0,4639 ; 0,4628 ; 0,4990$; and $\left.0,4510^{\prime \prime}\right)$ as well as the rather modest increase in alpha affected by the removal of the items $(0,0056 ; 0,0055 ; 0,0001$ and 0,006$)$, however, argued against deleting these items. Eighteen of the subscales returned Cronbach alpha values greater than 0,80 , with only the Inspiring followers towards ethical behaviour subscale falling marginally below this cutoff value $(0,79)$. The relatively high item homogeneity found for each sub-scale, as indicated by the Cronbach alpha values in Table 6 , is regarded as satisfactory.

\section{Dimensionality analysis}

Unrestricted principal component analyses with Varimax rotation were subsequently performed on each of the nineteen ELI subscales, each representing a facet of the multi-dimensional ethical leadership construct, to further evaluate the success with which each item accomplishes its intended function. The objective of these analyses furthermore was to confirm the uni-dimensionality of each sub-scale and to remove items with inadequate factor loadings (and rerun the item analysis) and/or split heterogeneous sub-scales into two or more homogenous subsets of items if necessary. In the latter case concomitant adjustments to the underlying ELI measurement model would have to be made and the item analysis repeated on the newly created subscales. Hulin, Drasgrow and Parsons 
(1983), however, caution that factor analysis as performed here on a matrix of product moment correlations might not be the most appropriate procedure for establishing the unidimensionality of a scale due to the danger of extracting artefact factors reflecting differences in item difficulty value or variance only. Descriptive statistics were therefore also calculated for the items of each subscale to examine the possibility of multiple factors appearing as an artefact of differential item characteristics like skewness (Schepers, 1992). The analyses were performed utilising SPSS 11.0 for Windows (2004). The eigenvalue-greater-than-unity rule of thumb was used to determine the number of factors to extract. All nineteen subscales passed the unidimensionality test. Factor loadings generally were satisfactory, varying between 0,565 and 0,870 with a median loading of 0,779 . Seventy five percent of the loadings exceeded 0,747. All four items flagged as suspect during the item analyses returned loading in excess of 0,60 , which on the one hand confirms their border-line status but simultaneously seems to justify the decision to retain these items. It should, nonetheless, be noted that for all subscales the single principle component solution failed to satisfactorily reproduce the observed correlation matrices. The results of the principle component analyses are summarized in Table 7.

The majority of items followed a significantly $(\mathrm{p}<0.05)$ negatively skewed and leptokurtic distribution. Due to the absence of negative items and the consistency in distributional form across items this did, however, not result in the emergence of artefact factors.

The results of the foregoing analyses seem to suggest that the ELI items generally do systematically reflect their designated latent ethical leadership dimension with reasonable success. Although no conclusive evidence in this regard was derived from the current data set, it is nonetheless assumed that the scales do reflect the intended latent variables. Results on the fit of the first-order measurement model reported below tend increase the confidence in this position. Expanding the measurement model into a fully fledged theory driven structural model and confronting these with the current data set via a series of confirmatory model fitting analyses utilizing Lisrel would, however, be needed to give significant credibility to this claim. The moderately high component loadings, the moderate percentage of the variance in the data explained by the single components, and the general inability of the single component solutions to reproduce the observed correlation matrices do, however, suggest that the ELI items generally also reflect a fair amount of non-relevant information.

\section{Variable type}

Structural equation modeling on the ELI in which each individual item serves as a manifest or indicator variable of the various latent leadership facets would have resulted in an extremely cumbersome and extensive exercise simply due to the number of items involved. The ordinal nature of the data would have required the calculation of the asymptotic covariance or asymptotic variance matrices which tend to demand large amounts of memory and processing time when the number of variables are large (Jöreskog \&
Sörbom, 1996a; 1996b). Consequently, two manifest variables were created from each sub-scale by calculating the unweighted average of the odd numbered items and the even numbered items of each scale. Apart from simplifying the logistics of fitting the model, the creation of two linear composite indicator variables for each latent variable has the added advantage of creating more reliable indicator variables (Nunnally, 1978). Marsh, Hau, Balla and Grayson (1998), on the other hand, report that solutions in confirmatory factor analysis tend to improve with increasing number of indicators per factor. The complexity of the comprehensive Lisrel model that would have resulted from treating the individual items as indicator variables swayed the decision towards parcelling. The composite indicator variables were treated as continuous variables. The analysis of the covariance matrix instead of the polychoric correlation matrix was therefore regarded as permissible (Jöreskog \& Sörbom, 1996a; 1996b; Mels, 2003).

\section{Univariate and multivariate normality}

The default method of estimation when fitting measurement models to continuous data (maximum likelihood), assumes multivariate normality. This is also true for generalized least squares (GLS) and full information maximum likelihood (FIML) as possible alternative estimation methods for structural equation modeling with continuous data (Mels, 2003). The inappropriate analysis of continuous non-normal variables in structural equation models can result in incorrect standard errors and chi-square estimates (Du Toit \& Du Toit, 2001; Mels, 2003). The univariate and multivariate normality of the composite indicator variables were consequently evaluated via PRELIS (Jöreskog \& Sörbom, 1996a). The null hypothesis of univariate normality had to be rejected in the case of all thirty-eight composite indicator variables, corroborating the skewness findings reported earlier. The results of the test for multivariate normality are given in Table 8 . The assumption of multivariate normality clearly is not tenable.

Two possible solutions to the lack of normality in the data were investigated. The first was to normalize the composite indicator variables. Although the PRELIS normalization had the effect of improving the symmetry and kurtosis of the indicator variable distributions, the null hypothesis of multivariate normality still had to be rejected. The dilemma is that normalizing occurs for each variable separately (Mels, 2003).

Since the normalization option failed to achieve multivariate normality, the use of an alternative method of estimation more suited to data not following a multivariate normal distribution was considered instead. This approach has the advantage over the use of normal scores that the solution need not be interpreted in terms of transformed values. Weighted least squares (WLS), diagonally weighted least squares (DWLS) and robust maximum likelihood (RML) are suggested to fit structural equation models to non-normal data (Du Toit \& Du Toit, 2001; Jöreskog, Sörbom, Du Toit, \& Du Toit, 2000; Mels, 2003). Mels (2003) recommends the use of robust maximum likelihood estimation if the assumption of a multivariate normal distribution does not hold. 
Table 6: Reliability of ELI sub-scale measures

\begin{tabular}{|c|c|c|c|c|c|c|}
\hline Subscale & Sample size (n) & Alpha & Mean & Variance & $\begin{array}{l}\text { Number of } \\
\text { items in final } \\
\text { subscale }\end{array}$ & $\begin{array}{l}\text { Number of } \\
\text { items deleted }\end{array}$ \\
\hline Environ & 249 & 0,82 & 20,17 & 12,63 & 5 & 0 \\
\hline Evision & 231 & 0,89 & 28,08 & 30,77 & 7 & 0 \\
\hline Butrust & 269 & 0,85 & 21,15 & 13,57 & 5 & 1 \\
\hline Artivis & 253 & 0,83 & 20,43 & 12,98 & 5 & 0 \\
\hline Costrat & 259 & 0,81 & 20,91 & 12,58 & 5 & 0 \\
\hline Enablel & 262 & 0,82 & 21,17 & 12,16 & 5 & 0 \\
\hline Enablef & 207 & 0,82 & 23,44 & 20,55 & 6 & 0 \\
\hline $\begin{array}{l}\text { Strusys } \\
\end{array}$ & 229 & 0,89 & 28,73 & 25,43 & 7 & 0 \\
\hline Ethcult & 266 & 0,81 & 17,07 & 8,99 & 4 & 0 \\
\hline Hointeg & 262 & 0,84 & 21,12 & 12,32 & 5 & 1 \\
\hline Actdeci & 271 & 0,83 & 21,26 & 11,84 & 5 & 0 \\
\hline Demsens & 247 & 0,85 & 24,27 & 18,31 & 6 & 0 \\
\hline Challen & 248 & 0,85 & 20,17 & 13,82 & 5 & 0 \\
\hline Inspire & 261 & 0,79 & 20,66 & 12,38 & 5 & 0 \\
\hline Intcoor & 252 & 0,85 & 20,22 & 14,15 & 5 & 0 \\
\hline Infstak & 262 & 0,81 & 20,97 & 11,92 & 5 & 0 \\
\hline $\begin{array}{l}\text { Planimp } \\
\end{array}$ & 241 & 0,85 & 20,12 & 14,76 & 5 & 0 \\
\hline Reviewe & 244 & 0,83 & 19,75 & 15,23 & 5 & 0 \\
\hline Rewarde & 223 & 0,82 & 20,13 & 13,25 & 5 & 0 \\
\hline
\end{tabular}

Table 7: Principle component analyses of ELI sub-scale measures

\begin{tabular}{l|c|c|c|c|c|c}
\hline \multicolumn{1}{c}{ Subscale } & Determinant & KMO & $\begin{array}{c}\text { \% Variance } \\
\text { explained }\end{array}$ & Max $\boldsymbol{A}$ & Min $\boldsymbol{\lambda}$ & $\begin{array}{c}\text { \% Residual } \mathbf{r}> \\
\mathbf{0 , 0 5}\end{array}$ \\
\hline Environ & 0,18 & 0,81 & 58,44 & 0,829 & 0,636 & 80 \\
\hline Evision & 0,02 & 0,89 & 61,87 & 0,844 & 0,708 & 71 \\
\hline Butrust & 0,13 & 0,83 & 62,46 & 0,847 & 0,740 & 70 \\
\hline Artivis & 0,11 & 0,81 & 61,79 & 0,870 & 0.629 & 70 \\
\hline Costrat & 0,18 & 0,81 & 58,76 & 0,816 & 0,735 & 80 \\
\hline Enablel & 0,19 & 0,84 & 58,89 & 0,832 & 0,710 & 90 \\
\hline Enablef & 0,08 & 0,88 & 60,29 & 0,826 & 0,730 & 80 \\
\hline Strusys & 0,05 & 0,90 & 64,67 & 0,842 & 0,747 & 66 \\
\hline Ethcult & 0,03 & 0,89 & 60,48 & 0,822 & 0,744 & 61 \\
\hline Hointeg & 0,25 & 0,78 & 64,49 & 0,847 & 0,749 & 100 \\
\hline Actdeci & 0,17 & 0,85 & 60,08 & 0,827 & 0,712 & 100 \\
\hline Demsens & 0,18 & 0,83 & 59,71 & 0,805 & 0,755 & 100 \\
\hline Challen & 0,09 & 0,86 & 58,30 & 0,805 & 0,646 & 73 \\
\hline Inspire & 0,23 & 0,83 & 56,13 & 0,831 & 0,693 & 90 \\
\hline Intcoor & 0,15 & 0,86 & 61,90 & 0,837 & 0,748 & 100 \\
\hline Infstak & 0,16 & 0,78 & 58,62 & 0,827 & 0,649 & 80 \\
\hline Planimp & 0,10 & 0,78 & 63,52 & 0,852 & 0,747 & 70 \\
\hline Reviewe & 0,18 & 0,82 & 59,01 & 0,810 & 0,725 & 80 \\
\hline Rewarde & 0,19 & 0,83 & 57,95 & 0,821 & 0,618 & 90 \\
\hline
\end{tabular}

Table 8: Test of multivariate normality for continuous variables

\begin{tabular}{|c|c|c|c|c|c|c|c|}
\hline & kewness & & & Kur & & Skewn & rtosis \\
\hline Value & Z-Score & P-Value & $\begin{array}{c}\text { Value } \\
\text {. }\end{array}$ & Z-Score & $\begin{array}{c}\text { P-Value } \\
0000\end{array}$ & Chi-Square & P-Value \\
\hline
\end{tabular}

\section{Evaluation of the primary measurement model}

The ELI was developed to measure the multifaceted leadership of ethics construct to which a specific constitutive meaning has been attached as shown in Table 1 . Operational denotations were explicitly and intentionally produced to reflect specific facets of this construct. Specific ELI items were written to function as homogenous stimulus sets to which raters would respond with behaviour which would be relatively uncontaminated behavioural expressions of specific latent leadership dimensions. The question that needs to be answered is to what extent this premeditated operational design did succeed in providing a comprehensive and uncontaminated empirical grasp on the construct as defined. Given the intention of the ELI to measure specific, previously defined leadership competencies by means of a specific operational architecture, a hypothesis testing, restricted, confirmatory approach was followed. In terms of this approach, specific structural assumptions are made with regards to the number of latent variables underlying the ELI, the relations among the latent variables, and the specific pattern of loadings of indicator variables on these latent variables (Ferrando \& Lorenzo-Seva, 2000; Jöreskog \& Sörbom, 1993). To the extent to which a measurement model reflecting these 
assumptions would fit empirical data poorly, the measurement intention of the researchers would have failed.

Structural equation modelling (SEM) was used to perform a confirmatory first-order factor analysis on the parcelled data set. The design and structure of the ELI implies a specific factor structure or measurement model. Given that two manifest variables were created from each sub-scale by calculating the unweighted average of the odd numbered items and the even numbered items of each scale, the measurement model underlying the ELI can be expressed as equation 1 .

$\mathrm{X}=\Lambda_{\mathrm{X}} \xi+\delta$

where:

- $\quad \mathrm{X}$ is a $38 \mathrm{x} 1$ column vector of observable indicator scores,

- $\Lambda_{\mathrm{X}}$ is a $38 \mathrm{x} 19$ matrix of factor loadings,

- $\quad \xi$ is a $19 \times 1$ column vector of first-order latent leadership facets, and

- $\quad \delta$ is a $38 \times 1$ column vector of unique/measurement error components comprising the combined effect on $\mathrm{X}$ of systematic non-relevant influences and random measurement error (Jöreskog \& Sörbom, 1993).

The measurement model implies two additional matrices. A symmetric 19x19 covariance/correlation matrix $\Phi$ contains the correlations between the latent leadership competencies. The ELI measurement model thus assumes the primary leadership of ethics factors to be correlated. A diagonal $19 \times 19$ matrix $\theta_{\delta}$ depicts the variance in the error terms associated with the indicator variables. The diagonal nature of the $\theta_{\delta}$ matrix implies that the error terms $\delta$ are assumed to be uncorrelated across the indicator variables.

LISREL 8.54 (Du Toit \& Du Toit, 2001; Jöreskog et al., 2000) was used to determine the fit of the model expressed as equation 1. The data was first read into PRELIS (Jöreskog \& Sörbom, 1996a) to compute covariance and asymptotic covariance matrices to serve as input for the LISREL analysis. The model fit was evaluated through an analysis of a covariance matrix due to the assumed continuous nature of the item parcels. Robust maximum likelihood estimation was used to estimate the parameters set free in the model due to the failure of the data to satisfy the multivariate normality assumption. The latent variables contained in the model as such have no inherent scale, and neither are the values expressed in a meaningful unit of measurement. In specifying the model, the scales of measurement of the latent variables were not specified by setting the factor loadings on the first observed variable to unity. Instead of defining the origin and unit of the latent variable scales in terms of observable reference variables, the latent variables were rather standardized (Jöreskog \& Sörbom, 1993; Jöreskog \& Sörbom, 1998). The unit of measurement thus becomes the standard deviation $\sigma_{\mathrm{i}}(\xi)$. In the case of ordinally scaled (in contrast to ratio scaled) observed variables, this option seems preferable since the scale and origin of the observed variables are then essentially arbitrary as well. All factor loadings of each latent leadership variable were set free to be estimated, but only with regards to its designated observed variables. All remaining elements of $\Lambda_{\mathrm{X}}$ were fixed at zero loadings to reflect the assumed factorial simplicity of the ELI items (Tabachnick \& Fidell, 1989). The elements of $\Phi$ and $\theta_{\delta}$ were treated by default as free.

\section{Model identification}

Model identification concerns the question whether the nature of the model and the data would permit the determination of unique estimates for the freed parameters in the model (MacCallum, 1995). The model depicted as equation 1 satisfies both the necessary conditions for identification. The first requirement of establishing a definite scale for each latent variable will be met by treating each latent variable as a $(0 ; 1)$ standardized variable (MacCallum, 1995). The second requirement is also met, namely that the number of model parameters that are set free to be estimated $(t=247)$ should be less than the number of non-redundant variance/covariance terms in the observed sample covariance matrix $([(p+q)(p+q+1)] / 2=741)^{3}$ (Diamantopoulos \& Siguaw, 2000).

\section{Assessing overall goodness-of-fit of the first- order measurement model}

An admissible final solution of parameter estimates for the ELI measurement model was obtained after 21 iterations. The full spectrum of indices provided by LISREL to assess the absolute and comparative fit of the proposed measurement model is presented in Table 9. No single measure of fit can provide a conclusive verdict on model fit (Bollen \& Long, 1993; Schumacker \& Lomax, 1996). Rather, an integrative judgement must be reached by considering the full spectrum of fit measures depicted in Table 9.

The Satorra-Bentler $\chi^{2}$ test statistic $(1114,53)$ is significant $(p<0,01)$, thus resulting in a rejection of the null hypothesis of exact model fit $\left(\mathrm{H}_{0}: \quad \Sigma=\Sigma(\theta)\right)$. The first-order measurement model is therefore not able to reproduce the observed covariance matrix to a degree of accuracy that could be explained in terms of sampling error only. Treating the chi-square statistic as a descriptive badness-offit measure by expressing the Satorra-Bentler $\chi^{2}$ estimate in terms of the degrees of freedom $\left(\chi^{2} / \mathrm{df}=2,26\right)$, however, suggests that the measurement model demonstrates acceptable fit to the data (Schumacker \& Lomax, 1996). Kelloway (1998), though, warns that the guidelines indicative of good fit (ratios between 2 and 5) have very little justification other than researcher's personal modelling experience and advises against a strong reliance on the normed chi square. The null hypothesis of exact model fit is, moreover, rather unrealistic. Browne and Cudeck (1993: 137) consequently argue:

\footnotetext{
${ }^{3} \mathrm{p}=$ the number of $\mathrm{y}$-variables; $\mathrm{q}=$ the number of $\mathrm{x}$-variables; in this case $\mathrm{p}=0$.
} 
Table 9: Goodness of fit statistics

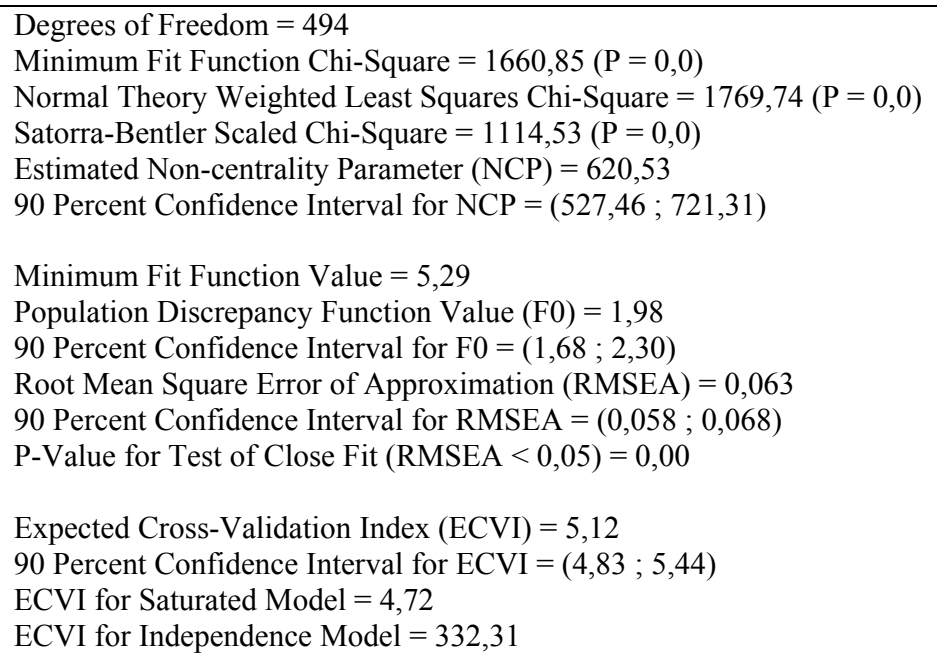

Chi-Square for Independence Model with 703 Degrees of Freedom $=104269,99$

Independence AIC = 104345,99

Model AIC $=1608,53$

Saturated AIC $=1482,00$

Independence CAIC $=104526,59$

Model CAIC $=2782,41$

Saturated CAIC $=5003,66$

Normed Fit Index $(\mathrm{NFI})=0,98$

Non-Normed Fit Index $(\mathrm{NNFI})=0,98$

Parsimony Normed Fit Index $(\mathrm{PNFI})=0,69$

Comparative Fit Index $(\mathrm{CFI})=0,99$

Incremental Fit Index (IFI) $=0,99$

Relative Fit Index $(\mathrm{RFI})=0,98$

Critical $N(\mathrm{CN})=108,77$

Root Mean Square Residual $(\mathrm{RMR})=0,022$

Standardized RMR $=0,035$

Goodness of Fit Index $(\mathrm{GFI})=0,77$

Adjusted Goodness of Fit Index (AGFI) $=0,66$

Parsimony Goodness of Fit Index $(\mathrm{PGFI})=0,51$

In applications of the analysis of covariance structures in the social sciences it is implausible that any model that we use is anything more than an approximation to reality. Since a null hypothesis that a model fits exactly in some population is known a priori to be false, it seems pointless even to try to test whether it is true.

If it were apriori assumed that the first-order measurement model only approximates the processes that operated in reality to create the observed covariance matrix, the $\chi^{2}$ test statistic will follow a non-central $\chi^{2}$ distribution with noncentrality parameter, $\lambda$ (Diamantopoulos $\&$ Siguaw, 2000). The estimated $\lambda$ value $(620,53)$ reflects the estimated discrepancy between the observed $\left(\Sigma_{0}\right)$ and estimated population covariance $\left(\tilde{\Sigma}_{0}\right)$ matrices (Diamantopoulos \& Siguaw, 2000).

The first-order measurement model was fitted by minimizing a fit function that compares the observed sample covariance matrix (S) to a reproduced sample covariance matrix $(\hat{\Sigma})$ derived from the model parameter estimates (Jöreskog \& Sörbom, 1993). An indication of the model fit achieved is provided by the extent to which the minimum fit function value $(5,29)$ approaches zero. The estimated population discrepancy function value $\left(\mathrm{F}_{0}\right)$ reflects the degree to which the observed population covariance matrix $\left(\Sigma_{0}\right)$ is estimated to differ from the reproduced population covariance matrix ( $\tilde{\Sigma}_{0}$ ) resulting from the model parameters minimising the selected discrepancy function when fitting the model on $\Sigma_{0}$ (Browne \& Cudeck, 1993). A point estimate of 1,98 is obtained for $F_{0}$ in this case with confidence limits of 1,68 and 2,30. Perfect exact fit would have been achieved if $\mathrm{F}_{0}$ had been zero because $\Sigma_{0}=\tilde{\Sigma}_{0}$. The root mean square error of approximation (RMSEA) expresses the error due to approximation per degree of freedom of the model. The RMSEA value of 0,063 indicates reasonable model fit, where reasonable fit is indicated by a value greater than 0.05 but less than 0,08 . The $90 \%$ confidence interval for RMSEA shown in Table $9(0,058-$ 0,068 ) indicates that the fit of the structural model could be regarded as good to reasonable. A test of close fit (in contrast to exact fit) is performed by LISREL by testing Ho: 
RMSEA $\leq 0,05$ against Ha: RMSEA $>0,05$. Table 9 further indicates that the obtained RMSEA value of 0,063 is significantly greater than the target value of 0,05 (i.e. $\mathrm{H}_{0}$ is rejected; $\mathrm{p}<0,05$ ), and since the confidence interval does not include the target value of 0.05 , a very good fit has not been achieved. In terms of the Browne and Cudeck (1993) guideline, however, the upper bound of the confidence interval suggests that reasonable fit has at least been obtained.

The expected cross-validation index (ECVI) expresses the difference between the reproduced sample covariance matrix $(\hat{\Sigma})$ derived from fitting the model on the sample at hand and the expected covariance matrix that would be obtained in an independent sample of the same size from the same population (Byrne, 1998; Diamantapolous \& Siguaw, 2000). It therefore focuses on the difference between $\hat{\Sigma}$ and $\Sigma$. Since the model ECVI $(5,12)$ is smaller than the value obtained for the independence model $(332,31)$ but larger than the ECVI value associated with the saturated model $(4,72)$, a model more closely resembling the saturated model seems to have a better chance of being replicated in a crossvalidation sample than the fitted model.

The assessment of parsimonious fit acknowledges that model fit can always be improved by adding more paths to the model and estimating more parameters until perfect fit is achieved in the form of a saturated or just-identified model with no degrees of freedom (Kelloway, 1998). The objective in model building is, however, to achieve satisfactory fit with as few model parameters as possible (Jöreskog \& Sörbom, 1993). The objective is therefore to find, in this sense, the most parsimonious model. Indices of parsimonious fit relate the benefit that accrues in terms of improved fit to the cost incurred (in terms of degrees of freedom lost) to affect the improvement in fit (Hair, Anderson, Tatham \& Black, 1995; Jöreskog \& Sörbom, 1993). The parsimonious normed fit index (PNFI $=0,69)$ and the parsimonious goodness-of-fit index $(\mathrm{PGFI}=0,51)$ shown in Table 9 approaches model fit from this perspective. Their meaningful use, however, necessitates a second, explicitly formulated and fitted model that contains a number of additional paths that can be theoretically justified so that the initial model is nested within the more elaborate model. In this case no such alternative model exists. The values for the Aiken information criterion (AIC $=1608,53$ ) shown in Table 9 suggest that the fitted structural model provides a more parsimonious fit than the independent/null model $(104345,99)$ but not the saturated model $(1482,00)$ since smaller values on these indices indicate a more parsimonious model (Kelloway, 1998). The values for the consistent Aiken information criterion (CAIC $=2782,41$ ), in contrast, suggest that the fitted structural model provides a more parsimonious fit than both the independent/null model $(104526,59)$ and the saturated model $(5003,66)$. This, in conjunction with the ECVI results, seems to suggest that the model still lacks one or more of influential paths.

Indices of comparative fit that use as a baseline an independence or null model, contrast the ability of the model to reproduce the observed covariance matrix with that of a model known apriori to fit the data poorly, namely one that postulates no paths between the variables in the model. The indices of comparative fit reported by LISREL and shown in Table 9 seem to indicate good model fit relative to that of the independence model. The normed fit index (NFI $=0,98)$, the non-normed fit index $(\mathrm{NNFI}=0,98)$, the comparative fit index $(\mathrm{CFI}=0,99)$, the incremental fit index $(\mathrm{IFI}=0,99)$, and the relative fit index $(\mathrm{RFI}=0,98)$ all can assume values between 0 and 1 with 0,90 generally considered indicative of a well-fitting model (Bentler, 1990; Bentler \& Bonett, 1980; Hair et al., 1995; Kelloway, 1998). The values of all of the aforementioned indices exceed the critical value of 0,90 thus indicating good comparative fit relative to the independence model.

The critical sample size statistic $(\mathrm{CN})$ reflects the size the sample that would have made the obtained minimum fit function $\chi^{2}$ statistic just significant at the 0,05 significance level. The estimated CN value $(108,77)$ falls noticeably short of the recommended threshold value of 200 , which is regarded as indicative of the model providing an adequate representation of the data (Diamantopoulos \& Siguaw, 2000). Hu and Bentler (1995), however, caution that this proposed threshold should be used with care.

The RMR $(0,022)$ and standardized RMR $(0,035)$ indicate good fit. Values of less than 0,05 on the latter index are regarded as indicative of a model that fits the data well (Kelloway, 1998).

The goodness-of-fit index (GFI), the adjusted goodness-offit index (AGFI) and the parsimony goodness-of-fit index (PGFI) reflect the success with which the reproduced sample covariance matrix recovered the observed sample covariance matrix (Diamantopoulos \& Siguaw, 2000; Schumacker \& Lomax, 1996). The AGFI adjusts the GFI for the degrees of freedom in the model whilst the PGFI makes an adjustment based on model complexity (Jöreskog \& Sörbom, 1993). The first two measures should be between zero and unity with values exceeding 0,9 indicating good fit to the data (Jöreskog \& Sörbom, 1993; Kelloway, 1998). Evaluating the fit of the model in terms of these two indices $(0,77$ and 0,66$)$ a relatively unfavourable conclusion on model fit emerges. Kelloway (1998), however, warns that these guidelines for the interpretation of GFI and AGFI are grounded in experience, are somewhat arbitrary and should therefore be used with some circumspection. According to Diamantopoulos and Siguaw (2000), acceptable values for the PGFI generally tend to be somewhat more conservative even when other indices indicate acceptable fit.

Integrating the results obtained on the full spectrum of fit statistics seems to suggest a good to reasonable fitting model that clearly outperforms the independence model but nonetheless fails to fully capture the true complexity of the processes underlying the ELI.

\section{Examination of residuals}

The stem-and-leaf plot of the standardized residuals (not shown) confirms the relative positive conclusion on model fit that was suggested by the fit statistics earlier. The distribution of standardized residuals appears to be 
distributed approximately symmetrical around a median standardized residual of zero. The smallest $(-1,54)$ and largest $(1,87)$ standardized residuals fall well within the 0,01 significance limits. The absence of large positive and negative residuals suggest that the observed covariance terms (741) in the observed sample covariance matrix (S) are being estimated reasonably well by the derived model parameter estimates. Reasonable model fit is moreover indicated by the relatively modest angular deviation of the standardized residuals for all pairs of observed variables from the $45^{\circ}$-reference line in the Q-plot, both in the upper and lower regions of the $\mathrm{X}$-axis.

\section{Model modification indices}

The fit of the proposed model depicted in equation 1 seems to be good to reasonable although not altogether without problems. The foregoing analysis of the standardized residuals did not suggest that the addition of one or more paths is desperately required to improve the fit of the model. This conclusion, however, does not agree with the inference derived from the values of the expected cross-validation index (ECVI) and the Aiken information criterion (AIC) earlier. The question of whether the addition of one or more paths would significantly improve the parsimonious fit of the model should therefore be examined more methodically by examining the modification indices calculated for the currently fixed parameters of the model. The modification indices calculated by LISREL estimate the decrease that should occur in the $\chi^{2}$ statistic if currently fixed parameters would be set free and the model re-estimated. Large modification index values $(>6,6349)$ thus indicate parameters that, if set free, would improve the fit of the model significantly $(\mathrm{p}<0.01)$ (Diamantopoulos \& Siguaw, 2000). Kelloway (1998) and Schumacker and Lomax (1996), however, caution that model modifications suggested by modification indices should be resisted unless such alterations to the model can be supported by clear and convincing theoretical justification.

Examination of the modification indices calculated for the $\Lambda_{\mathrm{X}}$ matrix indicates fifty-four additional paths that would significantly improve the fit of the ELI measurement model. The magnitude of the modification index values, taken in conjunction with the magnitude and sign of the standardized expected change values, would suggest that a number of the item parcels meant to be reflections of a single underlying latent variable could in fact be factorially complex. Noteworthy is the fact that the modification indices calculated for the $\Lambda_{\mathrm{X}}$ matrix suggest that the second Building trust item parcel (Butrust2) and the second Rewarding ethical contributions and behaviour item parcel (Rewarde2) could serve as indicators for an additional ten and nine latent leadership dimensions respectively. The same is, however, not true for the two associated parcels (Butrust1 and Rewarde1). This seems to suggest that the two parcels of each set assess subtly different aspects of Building trust and Rewarding ethical contributions and behaviour. The differences in theme, moreover, seem to be subtle enough to have escaped detection during the dimensionality analyses, possibly due to the rather insensitive nature of the eigenvalue-greater-than-one rule. The items included in the first Building trust item parcel seem to be related to demonstrated integrity (Engelbrecht, Scheps \& Theron, 2004), while the items included in the second Building trust item parcel (items 41 and 79) seem to be associated more with the content of the belief structure towards which consistency is displayed. The difference in theme reflected by the items included in the first and second Rewarding ethical contributions and behaviour item parcels (items 38 and 76) seem to be less apparent. Given the prominent and influential role of trust in leadership (Chamberlain, 2004; Krafft, Engelbrecht \& Theron, 2004) and the difficulty of simultaneously attending to rewarding and the remaining aspects of performance management (Spangenberg \& Theron, 1997), future research should probably follow up on these pointers.

Specific ELI items were explicitly and intentionally written to function as homogenous stimulus sets to which raters would respond with behaviour which would be relatively uncontaminated behavioural expressions of specific latent leadership dimensions. Subsequent results would suggest that the indicator variables do generally succeed in providing empirical grasp on the underlying latent variables they were meant to reflect (see Tables 10 and 11). The magnitude of the modification index values and the associated standardized expected change values would, however, suggest that many of them also provide information on latent variables they were not designed to reflect. The question is whether these capacities should be utilized by reflecting their contributions in the scoring key? This would largely depend on whether the proposed model modifications suggested by modification indices can be supported by clear and convincing theoretical justification (Diamantopoulos \& Siguaw, 2000; Kelloway, 1998). In the case of quite a few proposed model modifications it is possible to construct such justifications, although in some cases the explanation is somewhat more obscure. In addition, a significant number of the standardized expected change values associated with the proposed modifications to the $\Lambda_{\mathrm{X}}$ matrix are appropriately signed and of sufficient magnitude to consider freeing these elements. To justify freeing specific elements of $\Lambda_{\mathrm{X}}$ in terms of plausible explanations after the fact is, however, relatively easy, even in cases where no high modification index values were found. The more prudent and conservative option, at least for the moment, would be to remain faithful to the design intentions and not free any additional elements in $\Lambda_{\mathrm{X}}$. If the use of factorially complex items would be accepted as a basic design principle, then the architects of the ELI should re-examine in advance each of the current items and hypothesize specific additional non-zero loadings where applicable.

In addition, consideration should be given to the possibility that causal influence might exist between specific primary ethical leadership factors. Although this would, normatively speaking, probably constitute a somewhat novel approach to construct validation, it does not seem altogether unreasonable to argue that a relative lack of competence in one leadership competency might shackle performance in specific other competencies. It would therefore require the development of a fully-fledged structural model, rather than only a measurement model with correlated latent variables, 
to represent the assumed internal structure of the leadership of ethics domain.

Examination of the modification indices and the completely standardized expected parameter change associated with the fixed parameters in the $\Theta_{\varepsilon}$ matrix reveal 94 covariance terms that, if set free, would result in significant $(p<0.01)$ decreases in the $\chi^{2}$ measure. The expected magnitude of the completely standardized covariate estimates, however, hardly warrants seriously considering setting these parameters free. The expected completely standardized covariance between the measurement error terms associated with these $\Theta_{\varepsilon}$ elements are, with one exception, all smaller than $|0,11|$. The only exception being the proposed correlation between the measurement error terms associated with Rewardel and Enablell $(-0,15)$. This would suggest that the assumption of uncorrelated error terms remains largely tenable. In addition, there is no convincing theoretical rational to justify correlated measurement errors.

\section{Assessment of the first-order factor model}

The completely standardized factor loading matrix $\left(\Lambda_{\mathrm{X}}\right)$ (Table 10), reflecting the regression of $X_{j}$ on $\xi_{i}$, is used to evaluate the significance of the first-order factor loadings hypothesized by the proposed measurement model expressed as equation 1 .
The results depicted in Table 10 indicate that all proposed first-order factor loadings are significant $(p<0.05)$. The fit of the model should therefore deteriorate significantly if any of the existing paths in the measurement model would be pruned away by fixing the corresponding parameters in $\Lambda_{\mathrm{X}}$ to zero and thus effectively eliminating the items in question from their current sub-scales. None of the existing paths in the model thus appear to be redundant and all item parcels thus appear to significantly reflect the leadership dimension it was designed to denote. The completely standardized $\lambda$ parameter estimates reflect the average change in standard deviation units in a manifest variable $X$, directly resulting from a one standard deviation change in a first-order exogenous latent variable $\xi$ to which it has been linked, holding the effect of all other variables constant. Table 10 would moreover suggest that the indicator variables generally load quite high on the first-order factors to which they have been linked.

The proportion of item parcel variance that is explained by the latent variable it has been designated to reflect in terms of the measurement model (i.e. equation 1 ), is indicated by the squared multiple correlations for the observed indicator variables shown in Table 11.

Table 10: Completely standardized factor loading matrix $\left(\Lambda_{\mathrm{x}}\right)$

\begin{tabular}{|c|c|c|c|c|c|c|c|c|c|c|c|}
\hline \multicolumn{2}{|c|}{ Environ } & \multicolumn{2}{|c|}{ Evision } & \multicolumn{2}{|c|}{ Butrust } & \multicolumn{2}{|c|}{ Artivis } & \multicolumn{2}{|c|}{ Costrat } & \multicolumn{2}{|c|}{ Enablel } \\
\hline & 0,85 & & 0,89 & & 0,81 & & 0,91 & & 0,87 & & 0,86 \\
\hline \multirow[t]{3}{*}{ Environ1 } & $(0,05)$ & Evision 1 & $(0,05)$ & Butrust1 & $(0,07)$ & Artivis1 & $(0,05)$ & Costrat1 & $(0,05)$ & Enablel1 & $(0,06)$ \\
\hline & $13,89^{*}$ & & $14,83^{*}$ & & $9,32^{*}$ & & $14,81^{*}$ & & $12,21^{*}$ & & $10,52^{*}$ \\
\hline & 0,84 & & 0,86 & & 0,77 & & 0,89 & & 0,86 & & 0,85 \\
\hline \multirow[t]{2}{*}{ Environ2 } & $(0,05)$ & Evision2 & $(0,04)$ & Butrust2 & $(0,06)$ & Artivis2 & $(0,05)$ & Costrat2 & $(0,05)$ & Enablel2 & $(0,06)$ \\
\hline & $12,82^{*}$ & & $15,39^{*}$ & & $11,62^{*}$ & & $14,87^{*}$ & & $13,92^{*}$ & & $12,30^{*}$ \\
\hline \multicolumn{2}{|c|}{ Enablef } & \multicolumn{2}{|c|}{ Strusys } & \multicolumn{2}{|c|}{ Ethcult } & \multicolumn{2}{|c|}{ Hointeg } & \multicolumn{2}{|c|}{ Actdeci } & \multicolumn{2}{|c|}{ Demsens } \\
\hline \multirow{3}{*}{ Enablef1 } & 0,87 & \multirow{4}{*}{ Strusys1 } & 0,84 & \multirow{4}{*}{ Ethcult1 } & 0,91 & \multirow{4}{*}{ Hointeg1 } & 0,77 & \multirow{4}{*}{ Actdecil } & 0,89 & \multirow{3}{*}{ Demsens 1} & 0,87 \\
\hline & $(0,05)$ & & $(0,05)$ & & $(0,05)$ & & $(0,06)$ & & $(0,06)$ & & $(0,05)$ \\
\hline & $12,62^{*}$ & & $15,91^{*}$ & & $13,79^{*}$ & & $11,98^{*}$ & & $11,86^{*}$ & & $14,24^{*}$ \\
\hline \multirow[t]{3}{*}{ Enablef2 } & 0,90 & & 0.89 & & 0,91 & & 0,87 & & 0,81 & \multirow{3}{*}{ Demsens2 } & 0,79 \\
\hline & $(0,05)$ & \multirow[t]{2}{*}{ Strusys2 } & $(0,05)$ & Ethcult2 & $(0,05)$ & \multirow[t]{2}{*}{ Hointeg2 } & $(0,06)$ & Actdeci2 & $(0,06)$ & & $(0,06)$ \\
\hline & $12,08^{*}$ & & $15,18^{*}$ & & $14,76^{*}$ & & $11,25^{*}$ & & $9,85^{*}$ & & $9,03^{*^{\prime}}$ \\
\hline \multicolumn{2}{|c|}{ Challen } & \multicolumn{2}{|c|}{ Inspire } & \multicolumn{2}{|c|}{ Intcoor } & \multicolumn{2}{|c|}{ Infstak } & \multicolumn{2}{|c|}{ Planimp } & Revi & \\
\hline & 0,89 & & 0,83 & & 0,87 & & 0,88 & & 0,92 & & 0,85 \\
\hline Challen1 & $(0,05)$ & Inspire1 & $(0,06)$ & Intcoor1 & $(0,06)$ & Infstak1 & $(0,05)$ & Planimp1 & $(0,05)$ & Reviewe1 & $(0,05)$ \\
\hline & $14,43^{*}$ & & $11,12^{*}$ & & $11,26^{*}$ & & $14,39^{*}$ & & $15,70^{*}$ & & $13,71^{*}$ \\
\hline & 0,90 & & 0,80 & & 0,80 & & 0,81 & & 0,90 & & 0,77 \\
\hline Challen2 & $(0,05)$ & Inspire2 & $(0,06)$ & Intcoor2 & $(0,05)$ & Infstak2 & $(0,05)$ & Planimp2 & $(0,05)$ & Reviewe2 & $(0,06)$ \\
\hline & $13,34^{*}$ & & $11,78^{*}$ & & $13,09^{*}$ & & $12,31^{*}$ & & $14,23^{*}$ & & $11,72^{*}$ \\
\hline Rewa & & & & & & & & & & & \\
\hline & 0,90 & & & & & & & & & & \\
\hline Rewarde1 & $(0,04)$ & & & & & & & & & & \\
\hline & $17,03^{*}$ & & & & & & & & & & \\
\hline & 0,75 & & & & & & & & & & \\
\hline Rewarde2 & $(0,06)$ & & & & & & & & & & \\
\hline & $10,69^{*}$ & & & & & & & & & & \\
\hline
\end{tabular}

\footnotetext{
* t-values $>|1.96|$ indicate significant path coefficients; values in brackets represent standard error estimates
} 
Table 11: Squared multiple correlations for item parcels

\begin{tabular}{cccccccccc}
\hline Environ1 & Environ2 & Evision1 & Evision2 & Butrust1 & Butrust2 & Artivis1 & Artivis2 & Costrat1 & Costrat2 \\
0,73 & 0,71 & 0,79 & 0,74 & 0,65 & 0,60 & 0,83 & 0,79 & 0,76 & 0,74 \\
Enablel1 & Enablel2 & Enablef1 & Enablef2 & Strusys1 & Strusys2 & Ethcult1 & Ethcult2 & Hointeg1 & Hointeg2 \\
0,74 & 0,73 & 0,75 & 0,81 & 0,71 & 0,79 & 0,83 & 0,83 & 0,59 & 0,76 \\
Actdeci1 & Actdeci2 & Demsens1 & Demsens2 & Challen1 & Challen2 & Inspire1 & Inspire2 & Intcoor1 & Intcoor2 \\
0,79 & 0,65 & 0,76 & 0,63 & 0,79 & 0,81 & 0,69 & 0,63 & 0,75 & 0,63 \\
Infstak1 & Infstak2 & Planimp1 & Planimp2 & Reviewe1 & Reviewe2 & Rewarde1 & Rewarde2 & \\
0,78 & 0,66 & 0,86 & 0,81 & 0,72 & 0,58 & 0,80 & 0,56 & \\
\hline
\end{tabular}

The total variance in the $i^{\text {th }}$ item parcel $\left(\mathrm{X}_{\mathrm{i}}\right)$ could be decomposed into variance due to:

a) variance in the latent variable the item parcel was designed to reflect $\left(\xi_{\mathrm{j}}\right)$,

b) variance due to variance in other systematic latent effects the item parcel was not designed to reflect, and

c) variance due to random measurement error.

The latter two sources of variance in the item parcel are acknowledged in equation 1 through the measurement error term $\delta_{\mathrm{i}}$. The measurement error terms $\delta$ thus does not differentiate between systematic and random sources of error or non-relevant variance. The square of the completely standardized factor loading $\lambda$ (see Table10) could be interpreted as the proportion of systematic-relevant item parcel variance. The diagonal of the completely standardized theta-delta $\left(\theta_{\delta}\right)$ matrix reflects the proportion of non-relevant item parcel variance. The completely standardized error variance of the $i^{\text {th }}$ item parcel $\left(\theta_{\delta \mathrm{ii}}\right)$ thus consists of systematic non-relevant variance and random error variance. The values shown in Table 11 could therefore be interpreted as item parcel validity coefficients, $\rho\left(X_{i}, \xi_{j}\right)$. Since $\left(\lambda_{\mathrm{ij}}{ }^{2}+\theta_{\delta \mathrm{ii}}\right)$ are equal to unity in the completely standardized solution, the validity coefficients, $\rho\left(X_{i}, \xi_{j}\right)$ can be defined as follows:

$$
\begin{aligned}
\rho\left(\mathrm{X}_{\mathrm{i}}, \xi_{\mathrm{j}}\right) & =\sigma_{\text {systematic-relevant }}^{2}\left(\sigma_{\text {systematic-relevant }}^{2}+\sigma_{\text {non-relevant }}^{2}\right) \\
& =\lambda_{\mathrm{ij}}{ }^{2} /\left[\lambda_{\mathrm{ij}}{ }^{2}+\theta_{\delta \mathrm{ii}}\right] \\
& =1-\left(\theta_{\delta \mathrm{i}} /\left[\lambda_{\mathrm{ij}}{ }^{2}+\theta_{\delta \mathrm{ii}}\right]\right) \\
& =1-\theta_{\delta \mathrm{ii}} \\
& =\lambda_{\mathrm{ij}}{ }^{2}
\end{aligned}
$$

Since reliability could be defined as the extent to which variance in item parcels can be attributed to systematic sources, irrespective of whether the source of variance is relevant to the measurement intention or not, the values shown in Table 11 could simultaneously be interpreted as lower bound estimates of the item reliabilities $\rho_{\mathrm{ii}}$ (Diamantopoulos \& Siguaw, 2000; Jöreskog \& Sörbom, 1996b). The extent to which the true item reliabilities would be under-estimated would be determined by the extent to which $\delta_{\text {ii }}$ contains the effect of the systematic non-relevant latent influences. Given the results reported earlier on the modification indices calculated for $\Lambda_{\mathrm{X}}$, the under-estimation could be considerable.
In most cases the item parcels seem to provide relatively uncontaminated reflections of their designated latent dimensions. The success with which Hointeg1, Reviewe2 and Rewarde2 provide operational measures of the respective latent leadership dimensions they are meant to reflect is, however, not regarded as quite satisfactory.

The phi-matrix of correlations between the 19 latent leadership dimensions were also interpreted (not shown due to space limitations). The off-diagonal elements of the $\Phi$ matrix are the inter-leadership dimension correlations disattenuated for measurement error. These correlations are all significant $(\mathrm{p}<0,01)$ and all high to extremely high, thus suggesting the need to expand the model through the addition of a limited set of second-order factors. This is to a certain extent to be expected given the nature of the underlying multi-stage leadership model and the results obtained on the LBI (Spangenberg \& Theron, 2002; Theron $\&$ Spangenberg, 2004). The $\Phi$-matrix is moreover not positive definite with off-diagonal entries exceeding unity. These results are on the one hand rather disconcerting and tend to seriously challenge the discriminant validity of the first-order factors. On the other hand, the question arises whether the nature of the phi matrix is not an expression of the complexity of leadership (Cilliers, 1998) in the sense that the various dimensions comprising leadership are directly and indirectly causally influencing each other? It is noteworthy, given the modification results reported earlier, that the Building trust latent variable is especially affected by the presence of extreme, unacceptable correlations. It does not seem altogether unreasonable to argue that building trust in the leader and his vision is a crucial prerequisite for competence in quite a number of the other leadership competencies.

The conclusions reached by Ferrando and Lorenzo-Seva (2000) on performing confirmatory factor analysis on a set of factorially impure items, however, tend to mitigate the apparent severity of these results. They report that when analysing a set of factorially impure items with a typical CFA model:

... (a) the CFA solution is expected to fit badly .... (b) the parameter estimates of the loadings that are not fixed to zero are expected to be reasonably accurate although slightly upward biased; and (c) the inter-factor correlations are expected to be grossly overestimated (2000: 320). 


\section{Power assessment}

The statistical power associated with the tests of exact and close fit was estimated (Diamantopoulos \& Siguaw, 2000). To determine the power of these two hypothesis tests, a value of RMSEA $=0,05$ was assumed under the exact fit alternative hypothesis, while a value of RMSEA $=0,08$ was assumed under the close fit alternative hypothesis.

Power tables compiled by MacCallum, Browne and Sugawara (1996) only make provision for $\mathrm{v} \leq 100$ and $\mathrm{N} \leq 500$. A SPSS translation of the SAS syntax ${ }^{4}$ provided by MacCallum et al. (1996) was consequently used to derive power estimates for the tests of exact and close fit, given the effect sizes assumed above, a significance level $(\alpha)$ of 0,05 and a sample size of 315. The degrees of freedom ( $v$ ) in the model is $(1 / 2[(p+q][p+q+1]-t)=741-247=494$. Power values of 1,00 were obtained for both the tests of exact and close fit. Rejecting the null hypothesis of exact fit under the true condition of close fit thus is a certainty. Results indicate that even if the actual model fit would be as close as RMSEA $=0,03$, the probability of rejecting the null hypothesis of exact fit would still be as high as 0,989068 . The probability of rejecting the null hypothesis of close fit if the true model fit was mediocre is also unity. Even if the actual model fit would be a close as RMSEA $=0,06$, the probability of rejecting the null hypothesis of close fit would still be as high as 0,938511 . These power estimates, taken in conjunction with the decisions to reject the null hypotheses of exact and close fit, suggest that the conclusion of poor model fit could be contentious in that the tests of exact and close fit were highly sensitive to even trivial misspecifications in the model.

\section{Summary and managerial implications}

Ethical organizational unit behaviour is desired first and foremost because organizational behaviour conforming to ethical best practice is in and by it self highly valued. Moreover, organizational behaviour conforming to ethical best practice is highly valued, in part, because it is associated with outcomes or consequences beneficial to and valued by the individual leader, stakeholders, and the physical, social and economic environments in which the organizational unit operates. Consequently, such organizational unit behaviour can pay substantial dividends both in terms of preventing negative systemic responses and promoting positive systemic reactions. Seen from both these perspectives, a need therefore exists to increase the ethical behaviour standard demonstrated by the members of an organizational unit. ${ }^{5}$

\footnotetext{
${ }^{4}$ The help of Klay Martens of SPSS South Africa is gratefully acknowledged in finding an itterative procedure to determine the critical chi-square that would cut off an area alpha in the upper tail of the non-central chi-square distribution with $\mathrm{d}$ degrees of freedom and non-centrality parameter ncp.

${ }^{5}$ This raises the important question whether ethical unit behaviour should be reflected in the constitutive interpretation of unit performance, and consequently whether operational measures of unit performance like the Performance Index (Spangenberg \& Theron, 2004) should accommodate it as a separate dimension of unit performance?
}

The ethical behaviour standard demonstrated by the members of any given organizational unit is, however, not a chance event but rather is systematically determined by an intricate nomological network of latent variables. The ability to rationally and intentionally improve the ethical behaviour of the members of an organizational unit consequently depends on the extent to which the identity of the latent variables comprising this nomological network are known as well as the manner in which they combine to shape the ethical behaviour of unit members. Leadership characteristics and behaviour most probably play a vital role in such an explanatory model. It does not seem unreasonable to hold unit leaders responsible for the ethical quality of the behaviour of the members of the unit. The effect of leadership on the ethical behaviour standard demonstrated by the members of an organizational unit, however, most probably would not be direct, but rather would be mediated through a layer of leadership outcome latent variables. Ethical climate (Engelbrecht et al., 2004) and ethical culture are latent variables that quite possibly could mediate the effect of leadership on ethical member behaviour. Specific leadership behaviours (leadership competencies) are required to build an appropriate unit ethical climate and ethical culture and ultimately to achieve ethical unit behaviour.

Would a unit leader, acting in accordance with effective leadership prescribed by the LBI, while simultaneously complying with sound ethical standards, but without explicitly promoting an ethical vision, contribute towards building ethical unit climate and culture? Probably yes, because of the desire of followers to emulate their (transformational) leaders (Bass, 1985; Bass \& Avolio, 1993). This study would, however, want to argue that the building of ethical unit climate and ethical unit culture requires more than leaders who behave ethically. To purposefully build appropriate unit ethical climate and ethical culture and ultimately to achieve ethical unit behaviour additionally requires leaders of ethics. To achieve effective leadership of ethics, unit leaders have to register and be concerned about significant discrepancies between the ethical behaviour currently being displayed in the unit and an ethical behavioural norm reflecting prevailing ethical standards. Effective unit leaders of ethics have to have a clear ethical unit behaviour vision, which anticipates a reality dramatically different from traditional and conventional behavioural practices. Moreover, to achieve effective leadership of ethics, unit leaders have to be motivated to achieve the ethical vision. This results in the important and fascinating question of which attainments and dispositions move the leader of ethics to be disturbed by such ethical discrepancies and not to passively accept (like most others) the ethical shortcomings of unit members, but rather to be passionately committed to and active work towards the realization of a radically different ethical vision? The leader behaviours required to realize the ethical vision are essentially the same than those involved in the realization of any other vision. The fundamental position underlying the ELI is that the leadership competencies required to lead followers to ethical excellence in terms of the ethical vision are essentially the same as those involved in achieving unit performance excellence. 
The ELI, like the LBI, thus interprets leadership, as a complex, continuous process expressing itself in an extensive array of inter-dependent behavioural actions. The process essentially entails three sequentially linked phases (a) the development and selling of an appropriate yet challenging ethical vision for the unit, based on an assessment of the internal and external environment of the unit, (b) the preparation of the unit for the implementation of the ethical vision, and finally (c) the bold yet honest implementation of the vision by continually monitoring, revitalizing, fine-tuning and orchestrating a multitude of prerequisites for realizing the ethical unit vision. It should, however, be stressed that leadership is thereby not reduced to a finite, linearly strictly forward-moving process. Actually, the possibility raised earlier of identifying causal influences amongst first-order leadership dimensions, confesses that the existence of a complex (Cilliers, 1998), richly connected leadership structure is accepted as a basic premise.

From the perspective of the leadership model underlying the ELI, a total of 19 distinct latent leadership dimensions can be distinguished within the foregoing broad procedural structure, constituting the abstract themes common to bundles of leadership behavioural actions. Leading organizational units to build unit ethical climate and ethical culture and ultimately to achieve ethical unit behaviour thus represents a formidable task. Unit leaders very seldom would be proficient in all 19 of the aforementioned latent leadership dimensions. To improve leader effectiveness, and ultimately the ethical quality of unit member behaviour through leadership assessment and development, requires the identification of those latent leadership dimensions on which a leader performs relatively less well. The Ethical Leadership Inventory was developed specifically for the South African context to serve this purpose.

The results reported here provide reasonable support for the use of the 101-item ELI. The possibility of causal influences existing amongst specific first-order leadership dimensions, however, needs to be investigated. The current measurement model thus needs to be expanded into a fullyfledged ethical leadership structural model. Once satisfactory close fit has been achieved, the model will in addition have to be cross-validated (Diamantopoulos \& Siguaw, 2000) on an independent sample to provide sufficient evidence to really use the ELI with confidence for the development of leadership of ethics.

Given the assumed pivotal role of leadership in organizational unit performance, the nature of this presumed relationship should furthermore be captured in a comprehensive leadership-ethical unit behaviour structural model that would explain the manner in which the various latent leadership dimensions, mediated by (amongst others) ethical unit culture and ethical unit climate, affect the quality of ethical unit behaviour. At the same time, the question should be resolved whether ethical climate, ethical culture and ethical unit behaviour should be included in the constitutive definition of organizational unit performance (Spangenberg, \& Theron, 2004; Theron, Spangenberg, \& Henning, 2004; Theron, \& Spangenberg, 2002).

\section{References}

Andrews, K.R. 1989. 'Ethics in practice', Harvard Business Review, 89(5): 99 - 104.

Bass, B.M. 1985. Leadership and performance beyond expectations. New York: Free Press.

Bass, B.M. \& Avolio, B.J. 1993. 'Transformational leadership: A response to critiques.' In Chemmers, M. \& Ayman, R. (Eds.). Leadership theory and research perspectives and directions. Orlando: Academic Press.

Bass, B.M. \& Avolio, B. 1997. Manual for the multifactor leadership questionnaire. Redwood, CA: Mind Garden, Inc.

Bass, B.M. \& Steidlmeier, P. 1999. 'Ethics, character, and authentic transformational leadership behaviour', Leadership Quarterly, 10(2):181 - 217.

Bentler, P.M. 1990. 'Comparative fit indexes in structural models', Psychological Bulletin, 107(2):238 - 246.

Bentler, P.M. \& Bonett, D.G. 1980. 'Significance tests and goodness of fit in the analysis of covariance structures', Psychological Bulletin, 88(3):588 - 606.

Bollen, K.A. \& Long, J.S. 1993. Testing structural equation models. Newbury Park: Sage Publications.

Brickley, J.A., Smith, C.W. \& Zimmerman, J.L. 1994. 'Ethics, incentives, and organisational design', Journal of Applied Corporate Finance, 7(2):20-30.

Brien, A. 1998. 'Professional ethics and a culture of trust', Journal of Business Ethics, 17:391-409.

Browne, M.W. \& Cudeck, R. 1993. 'Alternative ways of assessing model fit', In Bollen, K.A. \& Long, J.S. (Eds.). Testing structural equation models. Newbury Park: Sage Publications.

Byrne, B.M. 1998. Structural equation modelling with LISREL, PRELIS, and SIMPLIS: Basic concepts, applications and programming. Mahwah, New Jersey: Lawrence Erlbaum Associates.

Chamberlain, L. 2004. 'The influence of transformational leadership on organisational citizenship behaviours'. Masters Thesis. University of Stellenbosch.

Cilliers, P. 1998. Complexity and post modernism: Understanding complex systems. London: Routledge.

Conger, J.A. \& Kanungo, R.N. 1998. Charismatic leadership in organisations. Thousand Oaks, CA: Sage Publications.

Cullen, J.B., Victor, B. \& Bronson, J.W. 1993. 'The Ethical Climate Questionnaire: An assessment of its development and validity', Psychological Reports, 73:667-674. 
Deshpande, S. P. 1996. 'Ethical climate and the link between success and ethical behaviour: an empirical investigation of a non-profit organisation', Journal of Business Ethics, 15:315-320.

Diamantopoulos, A. \& Siguaw, J.A. 2000. Introducing LISREL. London \& New Delhi: Sage Publications.

Du Toit, M. \& Du Toit, S.H.C. 2001. Interactive LISREL user's guide. Lincolnwood, IL: Scientific Software International.

Enderle, G. 1987. 'Some perspectives of managerial ethical leadership', Journal of Business Ethics, 6:657 - 663.

Engelbrecht, A.S., Scheps, A. \& Theron, C.C. 2004. 'The influence of ethical values on transformational leadership and ethical climate in organizations'. Paper presented at the Annual European Academy of Management (EURAM) Conference, University of St Andrews, Scotland.

Ferrando, P.J. \& Lorenzo-Seva, U. 2000. 'Unrestricted versus restricted factor analysis of multidimensional test items: some aspects of the problem and some suggestions', Psicológica, 21:301-323.

Hair, J.F., Anderson, R.E., Tatham, R.L. \& Black, W.C. 1995. Multivariate data analysis with readings. New Jersey: Prentice-Hall.

Hosmer, L.T. 1987. 'The institutionalisation of unethical behaviour', Journal of Business Ethics, 6:439 - 447.

House, R.J. 1995. 'Leadership in the twenty-first century'. In Howard, A. (Ed.). The changing nature of work. San Francisco: Jossey-Bass, pp. $411-450$.

Hu, L.T. \& Bentler, P.M. 1995. 'Evaluating model fit', In Hoyle, R.C. (Ed.). Structural equation modelling: Concepts, issues and applications. Thousand Oaks: Sage Publications.

Hulin, C.L., Drasgow, F. \& Parsons, C.K. 1983. Item response theory: Aapplication to psychological measurement. Homewood, Illinois: Dow Jones-Irwin.

Hummels, H. 1998. 'Organising ethics: A stakeholder debate', Journal of Business Ethics, 17(13):1403 - 1419.

Husted, B.W. 1998. 'Organisational justice and the management of stakeholder relations', Journal of Business Ethics. 17:643 - 651.

James, H.S. 2000. 'Reinforcing ethical decision making through organisational structure', Journal of Business Ethics, 28:43-58.

Jöreskog, K.G. \& Sörbom, D. 1993. LISREL 8: Structural equation modelling with SIMPLIS command language. Chicago: Scientific Software International.

Jöreskog, K.G. \& Sörbom, D. 1996a. PRELIS 2: User's reference guide. Chicago: Scientific Software International.
Jöreskog, K.G. \& Sörbom, D. 1996b. LISREL 8: User's reference guide. Chicago: Scientific Software International.

Jöreskog, K.G. \& Sörbom, D. 1998. Structural equation modelling with the SIMPLIS command language. Chicago: Scientific Software International.

Jöreskog, K.G., Sörbom, D., Du Toit, S. \& Du Toit, M. 2000. LISREL 8: New statistical features. Chicago: Scientific Software International.

Jose, A. \& Thibodeaux, M.A. 1999. 'Institutionalisation of ethics: The perspective of managers', Journal of Business Ethics, 22:125 - 140.

Kanungo, R.N. \& Medonca, M. 1996. Dimensions of ethical leadership. Thousand Oaks: Sage Publications.

Kelloway, E.K. 1998. Using LISREL for structural equation modelling; a researcher's guide. Thousand Oaks: Sage Publications.

King Committee on Corporate Governance. 2002. King report on corporate governance for South Africa. Johannesburg: Institute of Directors in South Africa.

Krafft, P, Engelbrecht, A.S. \& Theron, C.C. 2004. 'The influence of transformational and transactional leadership on dyadic trust relationships through perceptions of fairness', South African Journal of Industrial Psychology, 30(1):1018 .

Lindsay, R.M., Lindsay, L.M. \& Irvine, V.B. 1996. 'Instilling ethical behaviour in organisations: A survey of Canadian companies', Journal of Business Ethics, 15: 393 407.

MacCallum, R.C. 1995. 'Model specification: procedures, strategies and related issues'. In Hoyle, R.H. (Ed.). Structural equation modelling: Concepts, issues and applications. Thousand Oaks, California: Sage Publications.

MacCallum, R.C., Browne, M.W. \& Sugawara, H.M. 1996. 'Power analysis and determination of sample size for covariance structure modelling', Psychological Methods, 1(2):130-149.

Maignan, I. \& Ferrell, O.C. 2000. 'Measuring corporate citizenship in two countries: The case of the United States and France', Journal of Business Ethics, 23:283 - 297.

Marsh, H.W., Hau, K.T., Balla, J.R. \& Grayson, D. 1998. 'Is more ever too much? The number of indicators per factor in confirmatory factor analysis', Multivariate Behavioural Research, 33(2):181-220.

Mels, G. 2003. A workshop on structural equation modeling with LISREL 8.54 for Windows. Chicago: Scientific Software International.

Nunnally, J.C. 1978. Psychometric theory. McGraw-Hill: New York. 
O’Boyle, E.J. \& Dawson, L.E. Jr. 1992. 'The American Marketing Association code of ethics: Instruction for marketers', Journal of Business Ethics, 11:921 - 932.

Ohmann, O.A. 1989. 'Skyhooks'. In Andrews, K.R. (Ed.). Ethics in practice: managing the moral corporation (pp.5869). Boston: Harvard Business School Press.

Schein, E.H. 1992. Organizational culture and leadership. San Francisco: Jossey-Bass.

Schepers, J.M. 1992. Toetskonstruksie, teorie en praktyk. Johannesburg: RAU Drukpers.

Schumacker, R.E. \& Lomax, R.G. 1996. A beginner's guide to structural equation modeling. Mahaw, New Jersey: Lawrence Erlbaum Associates, Publishers.

Spangenberg, H.H. \& Theron, C.C. 1997. 'Developing a performance management audit questionnaire', South African Journal of Psychology, 27(3):143-50.

Spangenberg, H.H. \& Theron, C.C. 2002. 'Development of a uniquely South African leadership questionnaire', South African Journal of Psychology, 32(2):9-25.

Spangenberg, H.H. \& Theron, C.C. 2004. 'Development of a performance measurement questionnaire for assessing organisational work unit effectiveness', South African Journal of Industrial Psychology, 30(1):19-28.

'SPSS 11.0 for Windows'. 2004. SPSS Inc. http://www.spss.com/

Strong, K.C., Ringer, R.C. \& Taylor, S.A. 2001. 'The rules of stakeholder satisfaction', Journal of Business Ethics, 32:219-230.

Tabachnick, B.G. \& Fidell, L.S. 1989. Using multivariate statistics ( $2^{\text {nd }}$ Edition). New York: Harper Collins Publishers.

Theron, C.C. \& Spangenberg, H.H. 2002. 'Development of a performance measurement questionnaire for assessing organisational work unit effectiveness'. Paper presented at the Global Conference on Business and Economics, Paris.

Theron, C.C. \& Spangenberg, H.H. 2004. 'Towards a comprehensive leadership - unit performance structural model: The development of second-order factors for the leadership behaviour inventory (LBI)'. Manuscript accepted for publication in Management Dynamics.

Theron, C.C., Spangenberg, H.H. \& Henning, R. 2004. 'An elaboration of the internal structure of the unit performance construct as measured by the performance index (PI)', Management Dynamics, 13(2):35-52.

Trevino, L.K. \& Butterfield, K.D. 1998. 'The ethical context in organisations: Influences on employee attitudes and behaviours', Business Ethics Quarterly, 8:447-476.
Trevino, L.K. \& Nelson, K.A. 1995. Managing business ethics: Straight talk about how to do it right. New York: J Wiley \& Sons.

Trevino, L.K., Hartman, L.P. \& Brown, M. 2000. 'Moral person and moral manager: How executives develop a reputation for ethical leadership', California Management Review, 42(4):128-142.

Upchurch, R.S. \& Ruhland, S.K. 1996. 'The organisational bases of ethical work climates in lodging operations as perceived by general managers', Journal of Business Ethics, 15:1083-1097.

Vaicys, C., Barnett, T. \& Brown, G. 1996. 'An analysis of the factor structure of the Ethical Climate Questionnaire', Psychological Reports, 79:115-120.

Vardi, Y. 2001. 'The effects of organisational and ethical climates on misconduct at work', Journal of Business Ethics, 29:325-337.

Victor, B. \& Cullen, J.B. 1987. 'A theory and measure of ethical climate in organisations', Research in corporate Social Performances and Policy, 9:51-57.

Victor, B. \& Cullen, J.B. 1990. 'A theory and measure of ethical climate in organisations'. In Frederick, W.C. \& Preston, L.E. (Eds.). Business Ethics: research issues and empirical studies. Greenwich CT: JAI Press, pp. 77-97. 